\title{
The need and purpose of launching of a new journal Building up to Excellence
}

\author{
Pradhan RL
}

According to the Council of Science Editors, when starting a new journal, it should have an aim and also define the scope of the journal. Then the Editorial board is recruited, followed by sorting the finances and staff and design the journa and also decide on the number of issues in a year.

We at the NOAJ have tried to follow guidelines of the World Association of Medical Editors and tired our level best to bring this first issue. We have a specific aim in bringing out the journal and that is to publish the work we have been carrying out in Nepal at our various institutions. We know our audiences and that is you our colleagues from home and abroad. Since we are a specialty journal we know the limitation of the circulation and the number of articles that needs to be fit. We also have some role journals which are among the best in the world we try to emulate, and hopefully can reach their capacity in the future.

NOAJ is a new biomedical orthopaedic journal, committed to presenting highly significant research in various fields of Orthopaedic Surgery. The interdisciplinary nature of NOAJ requires that topics be of interest to experts in both clinical medicine and basic science research. Appropriate subject matter includes

1. The development and validation of disease models

2. The development of resources that promote the development of disease models

3. The use of established disease models to enhance our understanding of Orthopaedic diseases

The journal will provide a forum for the exchange of information and ideas across the entire spectrum of Orthopaedics research, including basic, translational and clinical research. NOAJ will publish original research reports that use model organisms either to elucidate the mechanisms underlying Orthopaedic diseases or to develop novel diagnostics and therapeutics. Model organisms of interest include Traumatology, Spinal surgery, Reconstructive and replacement surgery etc. NOAJ will promote communication between basic researchers using model topics, translational researches, and clinicians through the publication of original research articles, reviews, case reports, editorials and commentary with a common focus on the latest advancements in Orthopaedic surgery.

We know the climb to making NOAJ a journal of international stature is tough but with all the help from our fraternity, we will surely reach one day. Our journal will surely serve as a face and voice of Orthopaedic surgery in Nepal.

Based on the Short Course for Journal Editors May 1-2, Pittsburgh, PA, USA. 